\title{
Heterochromatin heterogeneity in Oedipoda germanica (Orthoptera) detected by in situ digestion with restriction endonucleases
}

\author{
C. Lopez-Fernandez \\ J. Gosalvez and \\ R. Mezzanotte*
}

Unidad de Genética. Departamento de Biología. CXV, Facultad de Ciencias, Universidad Autónoma de Madrid, 28049 Madrid, Spain.

* Istituto di Biologia Generale, Facoltá de Medicina e Chirurgia Via Ospedale 121, Cagliari, Italia.

Metaphase chromosomes of the grasshopper Oedipoda germanica were treated with AluI, Ddel, HaellI, HinfI, HpalI, MboI, MspI, Sau3A and TaqI restriction endonucleases (REs) and subsequently stained with Giemsa. C-banding and flurochromes of different base pair specificities (DA-DAPI or CMA ) were also used to localize and characterize the heterochromatin. Results indicate that (a) the large blocks of paracentromeric heterochromatin are heterogeneous within each chromosome. This heterogeneity is homogeneously distributed among almost all chromosomes of the genome. (b) The use of REs on fixed chromatin gives rise to a banding pattern not completely correlated with that obtained with C-banding; (c) heterochromatin associated with active NORs displays distinct characteristics: GC-rich (positive $\mathrm{CMA}_{3}$ ), high frequency of the restriction site CC GG, specific for MspI and HpaII, and low levels of methylation in the internal cytosine.

\section{INTRODUCTION}

Constitutive heterochromatin has been defined as permanently condensed blocks of chromatin located in discrete regions of homologous chromosomes of a given complement (Brown, 1966). Further analyses have corroborated its structural and functional differentiation when compared to the so-called euchromatin. Heterochromatic regions are commonly, though not always, composed of highly repeated DNA sequences, sometimes containing satellite DNA (for review see Brutlag, 1980). On the other hand, the protein component may also be particular since peculiar proteins are capable of binding peculiar DNA base sequences (Hsieh and Brutlag, 1979). In addition to these common features, distinct techniques of induced longitudinal differentiation of chromosomes (banding techniques) demonstrate both the quantitative and qualitative heterogeneity of constitutive heterochromatin. For instance, quantitative variations have been reported for heterochromatic regions of human chromosomes 1, 9, 16 and $\mathrm{Y}$ (Podugolnikova, 1979), while qualitative variations derive from the definition of different classes of heterochromatin within the same genome, as shown in Arcyptera tornosi where at least six types have been detected (Gosálvez et al., 1987).

Different cytogenetic tools have made possible the characterization of heterochromatic regions within a given genome. Standard C-banding is useful for localizing constitutive heterochromatic regions in both interphase and metaphase chromosomes (Sumner, 1972), while specific flurochromes such as 4'-6-diamidinophenylindole (DAPI), Hoechst 33258 , or chromomycin $\mathrm{A}_{3}$ $\left(\mathrm{CMA}_{3}\right)$ are capable of revealing the relative base pair richness (AT- or GC-rich DNA) of some C-bands on fixed metaphase chromosomes (Schweizer, 1976). Moreover, particular classes of GC- or AT-rich DNA can be studied by digesting fixed chromosomes with restriction endonucleases (REs) (Miller et al., 1984; Mezzanotte, 1986; Gosalvez et al., 1987). Extensive cleavage of a given repeated polynucleotide is produced whenever the recognition site of a certain $R E$ is present throughout the structure of such a DNA sequence and provokes pale or null staining in the chromosome areas containing such sequences. In contrast, failure of enzyme cleavage occurs whenever the repeated DNA does not contain the enzyme recognition site. In the latter case, the staining of these 
regions is similar to that shown by untreated chromosomes.

Most acridoid grasshoppers have appreciable quantities of heterochromatin in their complements. Results so far obtained by in situ hybridization techniques suggest that such regions display some molecular heterogeneity although they are quite homogeneous when occuppying similar regions of non-homologous chromosomes. For instance, in Atractomorpha similis all distal Cbands contain tandem blocks of highly repeated DNA, originating from the same family of sequences, which in turn are rather different from the C-bands localized near the centromeres (John et al., 1986). Such results are also found when scrutinizing base pair composition with fluorochromes (John et al., 1985).

In this paper we analyze the extent of heterochromatic molecular heterogeneity in the complement of $O$. germanica by in situ selective digestion with REs. Large paracentromeric C-positive blocks which characterize this grasshopper are studied to investigate (1) whether they react similarly to digestion with different REs, (2) the arrangement of DNA sequences within these blocks and (3) the equilocal distribution of the different heterochromatic fractions in the karyotype of this species.

\section{MATERIALS AND METHODS}

Meiotic chromosomes were obtained from males of O. germanica collected in Cala Domestica (Sardenya). Testes were removed and fixed in ethanol:acetic acid (3:1). Preparations of mitotic chromosomes were obtained from gonial mitosis. Single follicles of testes were squashed in a drop of 45 per cent acetic acid. The coverslip was removed with a razor blade after immersing the slides in liquid nitrogen. Slides were then air dried.

Fluorescence patterns were obtained using the methodology developed by Schweizer (1976, 1980). Photographs of $\mathrm{CMA}_{3}$ and DA-DAPI bands were taken on Valca F-22 film using a ZEISS photomicroscope. Silver impregnation was carried out according to the method developed by De la Vega and Gosálvez (1983). Nine restriction endonucleases (REs) (see specifications in table 1) were employed to digest fixed chromosomes in fresh air dried preparations. Tandem digestion with isoschizomers MboI-Sau3A and Hpall-MspI were used to test possible differences in the levels of methylation of the DNA. The slides were incubated with 100 microlitres of incubation buffer plus
Table 1 Different restriction endonucleases used in this work with the sequence cleaved. Dots over $\mathrm{C}$ and $\mathrm{A}$ indicate methylation of these bases

\begin{tabular}{lll}
\hline Enzyme & Sequence cleaved & Sequence not cleaved \\
\hline AluI & AG!CT & \\
DdeI & C!TNAG & \\
Haell & GG!CC & \\
Hinfl & G!ANTC & C!CGG \\
Hpall & C!CGG & !GÄTC \\
MboI & !GATC & C!CGG \\
MspI & C!CGG & \\
Sau3A & !GATC & \\
TaqI & T!CGA & \\
\hline
\end{tabular}

30 units of each enzyme. All REs, except TaqI, were incubated overnight at $37^{\circ} \mathrm{C}$. Incubation with TaqI was at $65^{\circ} \mathrm{C}$ overnight. The same times and temperatures were employed in control experiments using only the incubation buffer. Staining was performed with two per cent Giemsa in phosphate buffer for 15 to $30 \mathrm{~min}$.

\section{RESULTS}

The chromosome complement of $O$. germanica is $2 n=22+\mathrm{X}$ in the male and $2 n=22+\mathrm{XX}$ in the female. All chromosomes have apparently terminal centromeres and have been numbered in decreasing size order, according to their content of euchromatin, into two long $\left(\mathrm{L}_{1}-\mathrm{L}_{2}\right)$, seven medium $\left(\mathrm{M}_{3}-\mathrm{M}_{9}\right)$ and two short chromosome pairs $\left(\mathrm{S}_{10^{-}}\right.$ $\mathrm{S}_{11}$ ), the $\mathrm{X}$ being a medium sized chromosome.

The heterochromatin pattern, defined by $\mathrm{C}$ banding is basically restricted to the paracentromeric regions of all chromosomes in the form of large blocks (fig. 1(a), (b)). Autosome $L_{2}$ is an exception and shows pale blue chromatin in this region with small dots positively stained near the centromere.

A special chromosome pair, termed megameric, which displays early condensation in meiosis as compared to all remaining autosomes is present in many species of Orthoptera. In $O$. germanica this chromosome is the $\mathrm{M}_{9}$ and the $\mathrm{Ag}$-NOR technique localizes the only active nucleolar organizer region in the genome (fig. 1(e)) to an interstitial position on this chromosome (fig. $1(f))$.

DA/DAPI and $\mathrm{CMA}_{3}$ were used to differentiate mitotic chromosomes as well to allow some determination of the base composition of the DNA in C-bands (AT- or GC-rich DNA respectively). The paracentromeric C-bands showed bright fluorescence with DA-DAPI (fig. 1(d)). In addi- 


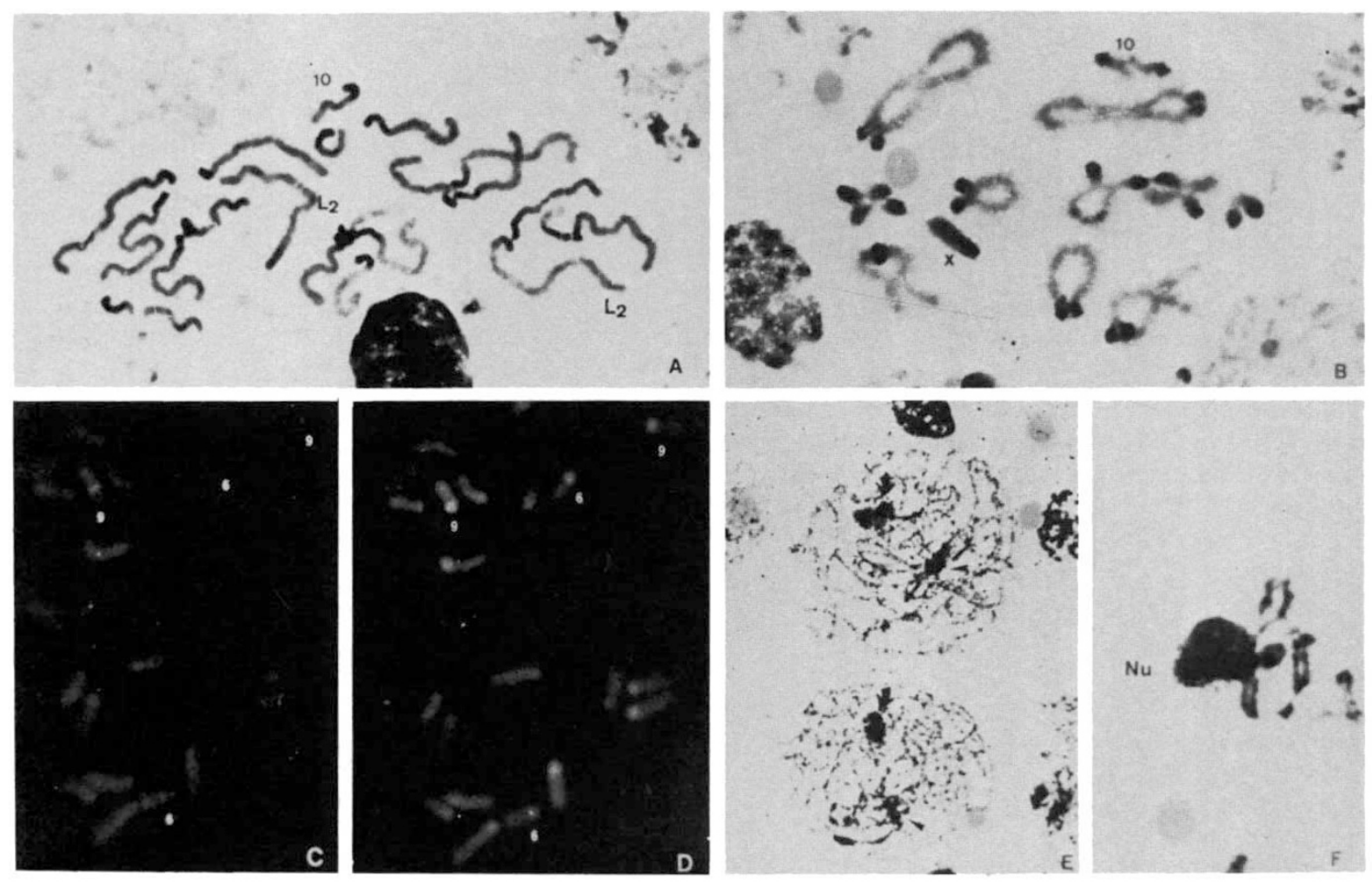

Figure 1 (a), (b) C-banded mitotic chromosomes (a) and diplotene (b) showing large blocks of heterochromatin in the paracentromeric regions. (c) Mitotic metaphase stained with $\mathrm{CMA}_{3}$. Note dull differential fluorescence in the centromere regions and more intense bands in chromosome 9 and 6. (d) Same plate stained with DA/DAPI. Differential staining is observed in the paracentromeric heterochromatin. (e), (f) NOR activity. Early meiotic prophase showing only a silver precipitate (arrow). Arrowhead corresponds to the X chromosome (e). Selected $M_{9}$ bivalent showing the localizataion of the single active NOR (f).

tion, there is a weak fluorescence with $\mathrm{CMA}_{3}$ in small centric regions of all chromosomes (fig. 1(c)). There is also a $\mathrm{CMA}_{3}$ positive band interstitially located on the megameric $\mathrm{M}_{9}$ chromosome which appears to be at the site of the active NOR (fig. $1(c))$. The fluorescence produced by this band is more intense than that found at the centromeres. Accordingly a gap is present in this region when chromosomes are stained with DA-DAPI (fig. 1(d)). One of the medium chromosomes, probably the $\mathrm{M}_{6}$, reveals a subdistal $\mathrm{CMA}_{3}$ band in addition to a centric and an interstitial band which seem to include a large DA-DAPI heterochromatic band (compare fig. 1(c) and 1(d)).

\section{Restriction enzymes}

In situ digestion of mitotic chromosomes with REs and subsequent staining with Giemsa produces linear bands which are different, at least in some chromosomes, from those obtained with Cbanding.
As the results obtained with different REs have different characteristics, we group our observations in two main sections: those regarding paracentromeric regions and those regarding chromosomes areas other than paracentromeric regions.

\section{Paracentromeric bands}

All REs used produce longitudinal differentiation in the complement of $O$. germanica but to different extents. AluI, DdeI, Mbol, Sau3A, (fig. 2) and TaqI (fig. 4(a), (c)) produce clearcut chromosome differentiation and high contrast between eu- and heteroch romatin. On the contrary, HaelII, HpaII, MspI and HinfI induce low contrast between euand heterochromatin (fig. 3(a), (c)).

Most REs produce paracentromeric bands which correspond to those obtained with Cbanding, although MboI and Sau3A reveal only a small positive band in that region of chromosome $\mathrm{S}_{10}$ (fig. 2(b), (d)). Interestingly, TaqI produces a reverse pattern in this chromosome with respect 

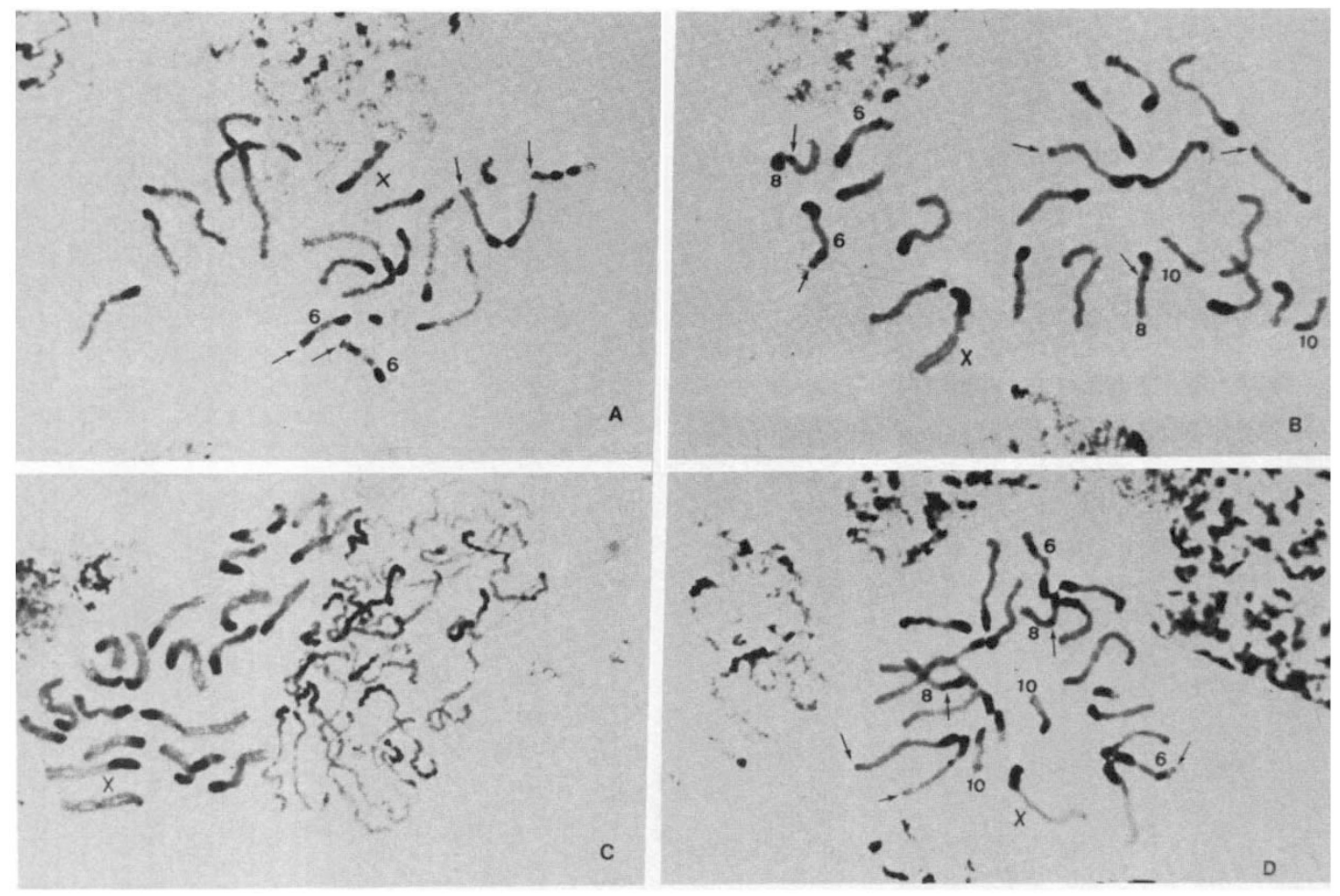

Figure 2 (a) Selective digestion with AluI. Arrows show positive bands not obtained with C-banding or fluorochromes. (b) Selective digestion with MboI. Arrowed chromosomes indicate bands not obtained with C-banding. Note the selective digestion produced in the paracentromeric heterochromatin of the $\mathrm{S}_{10}$ chromosome. The same pattern is obtained with the isoschizomer Sau3A (d). (c) Selective digestion with DdeI.

to that obtained with Mbol and Sau3A. That is, the only prominent paracentromeric positive band is located on chromosome $\mathrm{S}_{10}$ (fig. $4(\mathrm{c})$ ). Other REs which produce C-like bands such as AluI or DdeI show a similar pattern on $\mathrm{S}_{10}$ to that obtained with C-banding (fig. 6). Furthermore DA/DAPI and $\mathrm{CMA}_{3}$ show a bipartite nature to this region in terms of AT- or GC-rich DNA (fig. 6).

TaqI produces multiple positive bands interspersed with unstained regions within paracentromeric heterochromatin. This effect is clearly visible in early prophase chromosomes (fig. 4(a)). This differential intra-heterochromatic digestion is only partially observed in metaphase chromosomes (see multiple arrowed chromosomes in fig. $4(c))$. The visual effect produced by TaqI in metaphase chromosomes is a reduction in the size of the stained band in most of the chromosomes (fig. $4(c))$. All remaining enzymes induce homomorphic bands both in early prophase (compare fig. 4(a) with $4(\mathrm{~b})$ ) and in metaphase. A comparison of the general behaviour of the paracentromeric heterochromatin in metaphase chromosomes is shown in fig. 5 using the $\mathrm{L}_{1}$ chromosome. While C-bands and AluI localize constitutive heterochromatin, DA/DAPI and $\mathrm{CMA}_{3}$ reveal the bipartite nature of this zone. TaqI does not digest all the DA/DAPI or AluI positive bands. However, the TaqI band does not correspond to that obtained with $\mathrm{CMA}_{3}$.

\section{Non-paracentromeric bands}

Together with the paracentromeric bands described above several REs produce some positive bands as well as negative gaps in other chromosome regions. Hpall and $\mathrm{Mspl}$ induce unstained gaps in an interstitial region of $\mathrm{S}_{9}$ chromosome (fig. 3(c), (d), (e)). These gaps are particularly evident in condensed chromosomes and correspond to the chromatin associated to the active NOR detected with Ag-staining. This effect is also 

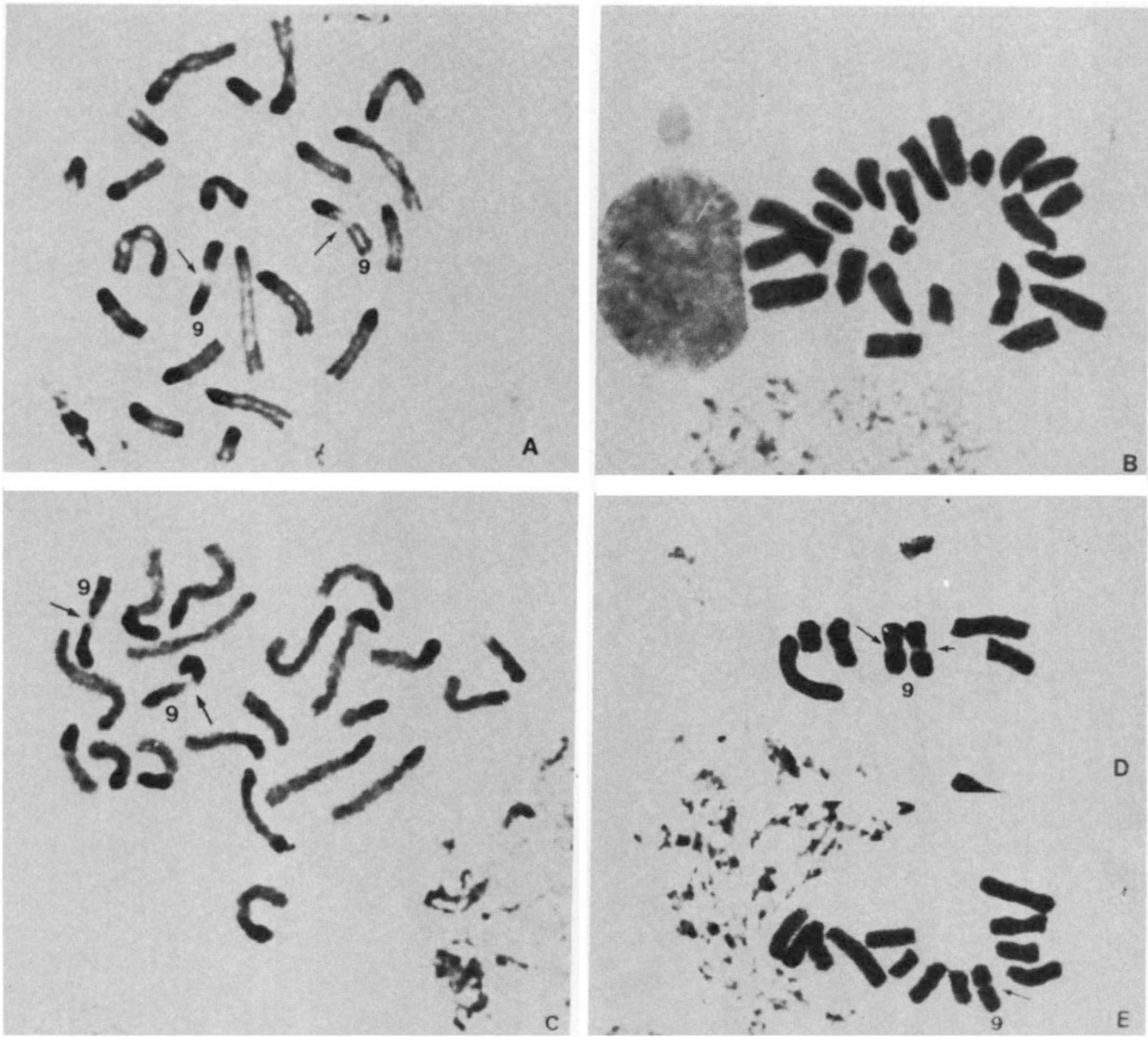

Figure 3 (a) Selective digestion with HaellI. Note a gap in chromosome $\mathbf{M}_{9}$. (b) Selective digestion with HinfT. No differentiation is produced. (c) Selective digestion with Mspl. Note a gap in chromosome $\mathbf{M}_{9}$. (d), (e) Selective digestion of metaphase chromosomes with MspI (d) and HpalI (e). Note the presence of gaps in chromosome $\mathbf{M}_{9}$.

observed in chromosomes digested with HaeIII (fig. 3(a)).

Distal positive dots in different chromosomes (see arrows in fig. 2(a)) are observed after digestion with AluI, Sau3A and Mbol (fig. 2(b), (d)). The same enzymes reveal a large interstitial band in chromosome $\mathbf{M}_{6}$ (fig. 2(a), (b), (d)). MboI and Sau3A, but not AluI, induce a positive interstitial band on chromosome $\mathbf{M}_{8}$ (fig. 2(b), (d)).

Finally, in slides treated with TaqI, discrete positive bands are obtained in the distal region of
$\mathrm{S}_{9}$ and the subparacentromeric region of chromosome $\mathrm{M}_{8}$ (fig. $4(\mathrm{c})$ ).

\section{DISCUSSION}

During recent years several reports on the characterization of heterochromatin in fixed chromosomes after digestion with REs have appeared (for review, see Babu and Verma, 1987). Our results (i) confirm the capability of REs to digest 

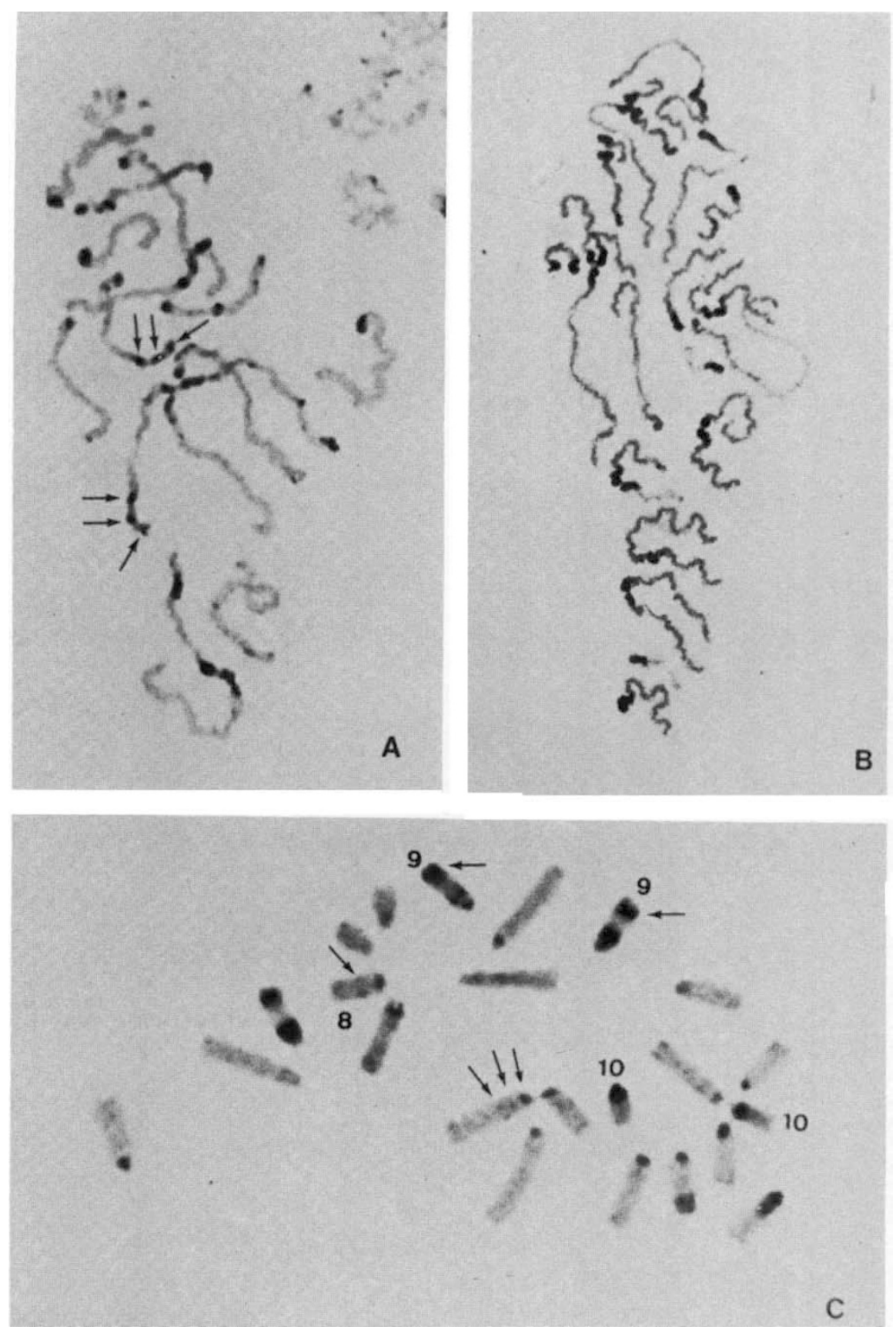

Figure 4 (a) Mitotic prophase digested with Taql. Arrows indicate the intra-heterochromatin banding produced by a differential digestion. (b) Mitotic prophase digested with AluI. Note homogeneous staining in all the centromere regions. (c) Metaphase plate digested with TaqI. The digestion of the paracentromeric heterochromating produces diminished staining in those regions. Arrows on numbered chromosomes indicate bands not obtained with C-banding. Arrows on unnumbered chromosomes indicate the metaphase visualization of the intra-heterochromatic digestion. 


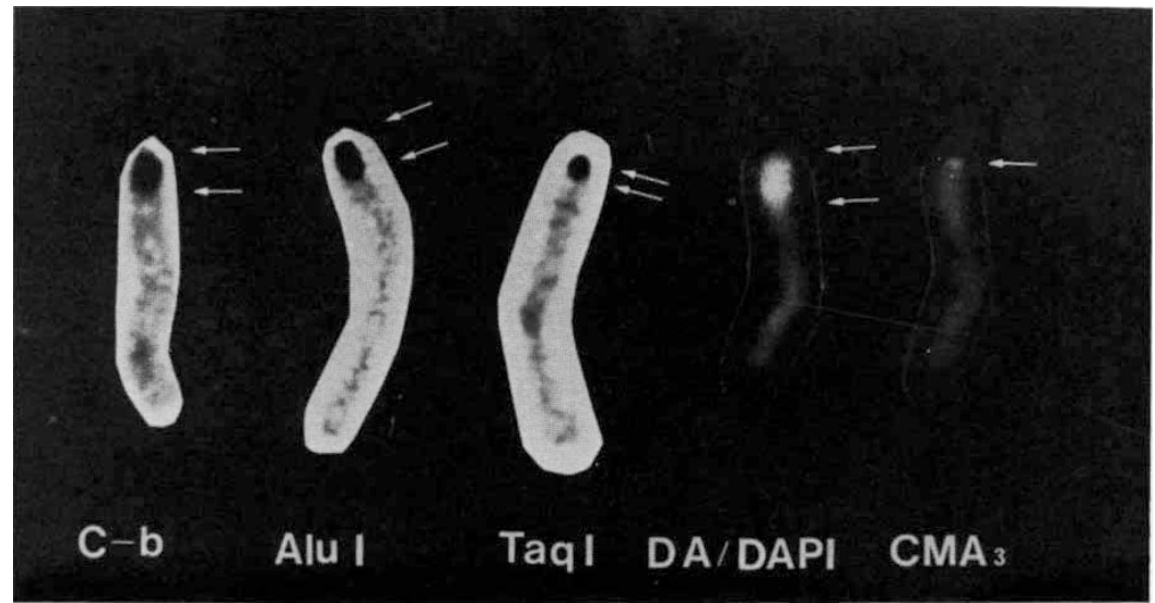

Figure 5 Selected $\mathrm{L}_{1}$ chromosomes to show the differential reaction of the heterochromatin to different treatments. Arrows indicate the extent of the differential staining.

the chromatin whatever its characteristics of condensation, (ii) reveal the existence of certain chromosome regions possibly containing repetitive DNA sequences though they are not detected with conventional banding techniques and (iii) uncover the heterogeneity of equilocally distributed heterochromatin.

Data so far obtained indicate that some REs are able to selectively digest chromosomal DNA regardless of the accessibility of the target, i.e., REs are able to recognize and cut specific targets whatever the nature of chromatin compactness. For instance, all mouse centromeres and human paracentromeric areas of chromosomes 1, 9 and 16 are characterized as highly condensed chromatin (heterochromatin) containing satellite DNA sequences. In both cases these regions result digested by enzymes such as Bst NI and HinfI, respectively (Southern, 1975; Miller et al., 1984; Bianchi et al., 1985). Such specific efficiency is shown in $O$. germanica by the different patterns of bands obtained on highly packed paracentromeric heterochromatin with REs such as AluI or TaqI, but is also supported by the production of a unique and specific band on $\mathrm{M}_{8}$ chromosome after the treatment with the isoschizomeres MboI

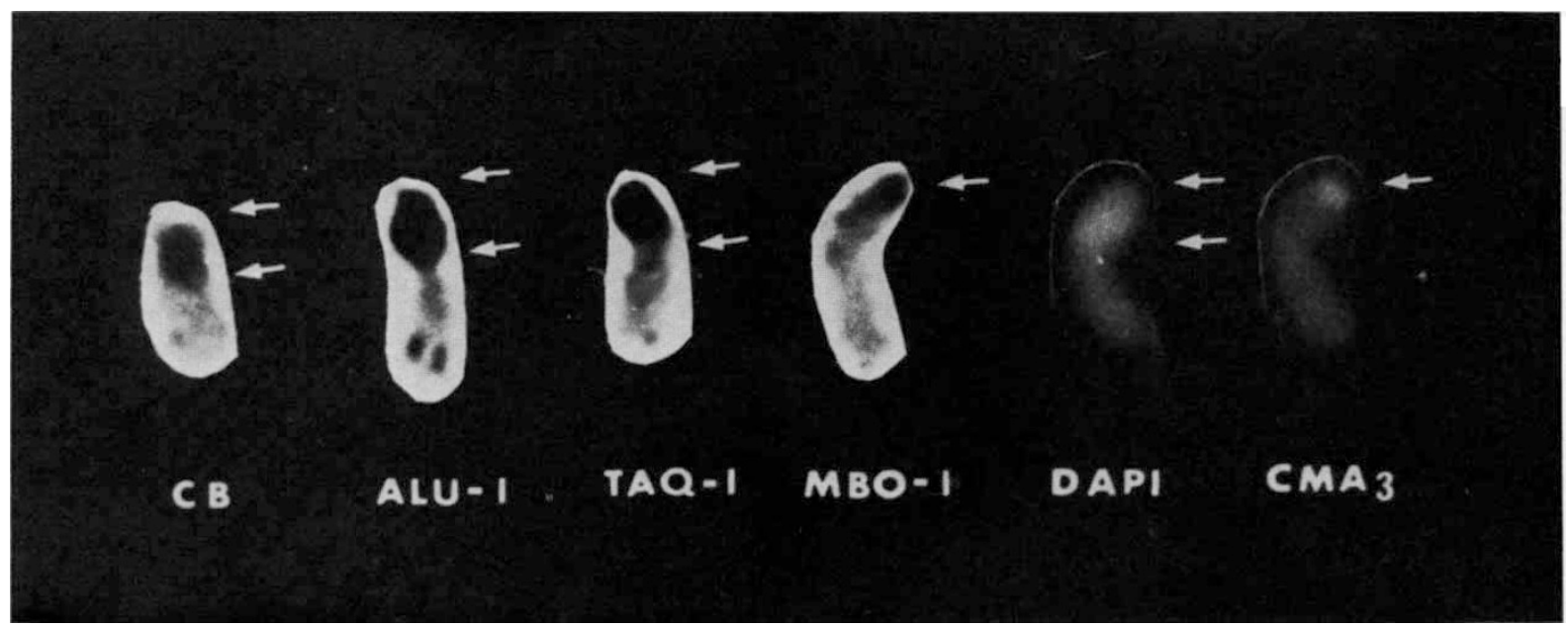

Figure 6 Selected $\mathrm{S}_{10}$ chromosomes to show the differential reaction of the heterochromatin to different treatments. Arrows indicate the extent of the differential staining. 
or Sau 3A. All these facts indicate that REs are capable of acting on chromatin whatever its structure, despite the presumable differences in molecular structure of distinct enzymes with identical targets. The same arguments apply to other enzymes which cut identical or similar targets (HaeIII, MspI and HpaII, in our case). In fact the gap produced by MspI and HpaII in the NORassociated $\mathrm{CMA}_{3}$ band suggests two important characteristics of this particular chromosome region: (i) the massive presence of $\mathrm{CC}$ GG restriction sites which agrees with the high GC richness of the DNA according to the strong positive fluorescence obtained with $\mathrm{CMA}_{3}$ and (ii) the absence or minor incidence of methylated cytosines (both internal and external since both REs produce the same banding pattern).

Constitutive heterochromatin is usually related to C-banded chromosome regions which are usually those differentially fluorescing with basepair specific dyes (John et al., 1985; Schmid et al., 1986). In general, this extends to the banding patterns obtained with certain REs (Lloyd and Thorgaard, 1988). However, as reported in the grasshopper A. tornosi (Gosalvez et al., 1987), complete correspondence between C-bands and chromosomal areas differentially reacting to the RE treatment is not always found. In $O$. germanica, as in A. tornosi, digestion with Alul induces telomeric dots in some chromosomes. These regions do not show C-bands or bright fluorescence. The interstitial band of chromosome $\mathrm{M}_{8}$ is not $\mathrm{C}$-banded and is only observable when digesting chromosomes with Sau3A or Mbol. These results confirm that C-bands do not necessarily coincide with REbands and also suggest that repetitive DNAs sequences are probably included in those bands.

The "equilocal distribution of heterochromatin" hypothesis predicts that certain chromosome regions within all the members of a given complement tend to accumulate this kind of chromatin (Heitz, 1933; Schweizer and Loidl, 1987). This has been confirmed by several reports that also demonstrate the molecular heterogeneity of heterochromatin located in different chromosome regions (Schweizer and Ehrendorfer, 1983; John et al., 1985). Our results in O. germanica show homogeneously C-banded large paracentromeric heterochromatic blocks within each chromosome. However specific fluorochromes reveal a bipartite structure of these blocks (heterogeneity). Similarly TaqI produces an interspersed pattern of positive and dull bands within these C-bands in most of the chromosomes. This arrangement suggests that repeated sequences are equilocated, in general, in paracentric heterochromatin and clustered in adjacent but not contiguous blocks. On the contrary, the homogeneous positive staining obtained in the same regions with enzymes such as AluI, DdeI, Sau3A, MboI and HaeIII indicates the absence of other restriction sites in the different subunits forming the heterochromatic blocks, at least with sufficient frequency to produce removable DNA fragments.

These equilocal patterns of heterochromatin also have some remarkable exceptions. For instance, Sau3A and MboI produce massive digestion of the paracentromeric heterochromatin of chromosome $\mathrm{S}_{10}$ probably due to the high frequency of the specific target which permits massive cleavage and removal of the DNA. The differential arrangements of the chromatin in this paracentromeric heterochromatin is reinforced by the homogeneous staining obtained after the digestion with TaqI. The restriction sites in this zone are clearly different in number and position to those found in other chromosomes.

Acknowledgements We thank Dr C. Garcia de la Vega for his very helpful comments and Dr J. L. Bella for technical assistance. This research was partially supported by CICYT (PB 86-0106) and Acciones Integradas Hispano-Italianas (1988).

\section{REFERENCES}

BABU, A. AN1) VERMA, R. S. 1987. Chromosome structure: Euchromatin and Heterochromatin. Int. Rev. Cytol., 108, $1-49$.

BIANCHI, M. S., BIANCHI, N. O., PANTELIAS, G. E. AND WOLFF, s. 1985. The mechanisms and pattern of banding induced by restriction endonucleases in human chromosomes. Chromosoma, 91, 131-136.

BROWN, S. W. 1966. Heterochromatin. Science, 151, 417-425.

BRUTLAG, D. L. 1980. Molecular arrangement and evolution of heterochromatic DNA. Ann. Rev. Genet. 14, 121-144.

DE LA VEGA, C. AND GOSÁlveZ, J. 1983. Differential NOR activity in two acridoid species. Biol. Zbl., 102, 545-549.

GosÁlveZ, 3., BELLA, 3. L., LÓPEZ-FERNÁNDEZ, C. AND MEZZANOTTE, R. 1987. Correlation between constitutive heterochromatin and restriction enzyme resistant chromatin in Arcyptera tornosi (Orthoptera). Heredity, 59 , $173-180$.

HEITZ, F. 1933. Die somatische heteropyknose bei Drosophila melanogaster und ihre genetische bedeutung. Z. Zellforsch, $20,417-425$.

HSIEH, T. AND BRUTLAG, D. L. 1979. A protein that preferentially binds Drosophila satellite DNA. Proc. Natl. Acad. Sci. USA, 76, 726-730.

JOHN, B., APPElS, R. AND CONTRERAS, N. 1986. Population cytogenetics of Atractomorpha similis. II. Molecular characterization of the distal C-band polymorphisms. Chromosoma, 94, 45-58. 
JOHN, B., KING, M., SCHWEIZER, D. AND MENDELAK, M. 1985. Equilocality of heterochromatin distribution and heterochromatin heterogeneity in acridid grasshoppers. Chromosoma, 91, 185-200.

LLOYD, M. AND THORGAARD, G. H. 1988. Restriction endonuclease banding of rainbow trout chromosomes. Chromosoma, 96, 171-177.

MEZZANOTTE, R. 1986. The selective digestion of polytene and mitotic chromosomes of Drosophila melanogaster by the AluI and HaeIII restriction endonucleases. Chromosoma, 93, 249-255.

Miller, D. A., Gosden, J. R., hastie, N. D. AND EVANS, H. J. 1984. Mechanism of endonuclease banding of chromosomes. Exp. Cell. Res., 155, 294-298.

PODUGOLNIKOVA, O. A. 1979. The quantitative analysis of polymorphism of human chromosomes $1,9,16$ and Y. III. Study of relationships of $\mathrm{C}$ segments length in individual karyotypes. Hum. Genet., 49, 261-268.

SCHMID, M., HAAF, T., OTT, G., SCHERES, J. M. J. C. AND WENING, J. A. B. 1986. Heterochromatin in the chromosomes of gorilla: characterization with distamycin A/DAPI, D287/170, chromomycin $\mathrm{A}_{3}$, quinacrine and 5azacytidine. Cytogenet. Cell. Genet., 41, 71-82.
SCHWEIZER, D. 1976. Reverse fluorescent chromosome banding with chromomycin and DAPI. Chromosoma, 58, 307324.

SCHWEIZER, D. 1980. Simultaneous fluorescent staining of $R$ bands specific heterochromatic regions (DA-DAPI bands) in human chromosomes. Cytogenet. Cell. Genet., 27, 190 193.

SCHWEIZER, D. AND LOIDL, J. 1987. A model for heterochromatin dispersion and the evolution of C-bands patterns. Chromosome Today, Vol. 9, Allen and Unwin. London, pp. 61-79.

SCHWEIZER, D. AND EHRENDORFER, F. 1983. Evolution of $\mathrm{C}$-band patterns in Asteraceae-Anthemidae. Biol. Zbl., 102, 637-655.

SOUTHERN, E. M. 1975. Long periodicities in mouse satellite DNA J. Mol. Biol., 94, 51-69.

SUMNER, A. T. 1972. A simple technique for demonstrating centromeric heterochromatin. Exp. Cell. Res., 75, 304-306. 